\title{
Power-like delay in time inhomogeneous Fisher-KPP equations
}

\author{
James Nolen* Jean-Michel Roquejoffre ${ }^{\dagger} \quad$ Lenya Ryzhik ${ }^{\ddagger}$
}

June 24, 2013

\begin{abstract}
We consider solutions of the KPP equation with a time-dependent diffusivity of the form $\sigma(t / T)$. For an initial condition that is compactly supported, we show that when $\sigma(s)$ is increasing in time the front position at time $T$ is $X(T)=c_{*} T-\bar{\nu} T^{1 / 3}+O(\log T)$. That is, $X(T)$ lags behind the linear front by an amount that is algebraic in $T$, not by the Bramson correction $(3 / 2) \log T$ as in the uniform medium. This refines a result by Fang and Zeitouni.
\end{abstract}

\section{Introduction}

The long time behavior of the solutions of the Fisher-KPP equation

$$
u_{t}=u_{x x}+f(u),
$$

has been studied since the original works by Fisher [6], and Kolmogorov, Petrovskii and Piskunov [12] that both appeared in 1937. The nonlinear function $f(u)$ is assumed to satisfy

$$
f(u)>0 \text { for } u \in(0,1), f(0)=f(1)=0 \text { and } f(u) \leq f^{\prime}(0) u \text { for } u \in[0,1] .
$$

Like other reaction-diffusion equations, this equation admits traveling wave solutions: for any $c \geq$ $c_{*}=2 \sqrt{f^{\prime}(0)}$ there exists a profile $U^{c}(x)$ that satisfies

$$
-c U_{x}^{c}=U_{x x}^{c}+f\left(U^{c}\right), \quad U^{c}(-\infty)=1, \quad U^{c}(+\infty)=0 .
$$

If the initial data for (1.1) is front-like, meaning that $0 \leq u(0, x) \leq 1, u(0, x)=0$ for $x>L$, for some $L \in \mathbb{R}$, and

$$
\liminf _{x \rightarrow-\infty} u(0, x)>0,
$$

then the solution at a later time will resemble a traveling wave in a shifted reference frame. An interesting problem is to determine the position of the front at large times. Let us define the front position as

$$
X(t)=\sup \{x: u(t, x)=1 / 2\} .
$$

Bramson has shown in his seminal papers $[1,2]$ that $X(t)$ has the asymptotics

$$
X(t)=c_{*} t-\frac{3}{2 \lambda_{*}} \log t+x_{0}+o(1), \text { as } t \rightarrow+\infty .
$$

\footnotetext{
*Department of Mathematics, Duke University, Durham, NC 27708, USA; nolen@math.duke.edu

${ }^{\dagger}$ Institut de Mathématiques (UMR CNRS 5219), Université Paul Sabatier, 118 route de Narbonne, 31062 Toulouse cedex, France; jean-michel.roquejoffre@math.univ-toulouse.fr

${ }^{\ddagger}$ Department of Mathematics, Stanford University, Stanford CA, 94305, USA; ryzhik@math.stanford.edu
} 
Here, $x_{0}$ is a constant shift that depends on the initial data, and $\lambda_{*}$ is the exponential decay rate for the critical traveling wave:

$$
U^{c_{*}}(x) \sim x e^{-\lambda_{*} x}, \text { as } x \rightarrow+\infty .
$$

Bramson's proof of (1.5) was probabilistic. In [13, 16], versions of Bramson's result were proved using the intersection number of the solution, a PDE technique which was used also in the original KPP paper [12]. An alternative probabilistic proof of Bramson's correction was recently given by Roberts [15], and an extension to higher dimensions was done by Gärtner [9]. A simple proof of a weaker version of (1.5) was presented recently in [10]:

$$
X(t)=c_{*} t-\frac{3}{2 \lambda_{*}} \log t+O(1), \text { as } t \rightarrow+\infty .
$$

It was extended to spatially periodic media in [11]. The main observation of $[10,11]$ is very simple and has been used already by Gärtner in the probabilistic context: solutions of the nonlinear equation (1.1) behave very similarly to those of the linear equation

$$
w_{t}=w_{x x}+f^{\prime}(0) w,
$$

with the Dirichlet boundary condition at a moving boundary: $w(t, Y(t))=0$. The idea is that if we choose the boundary $Y(t)$ "too far to the left" then $w(t, x)$ will grow exponentially in time, while if we choose $Y(t)$ "too far to the right" then $w(t, x)$ will decay in time. It turns out that if we choose $Y(t)$ so that $w(t, x)$ stays $O(1)$ as $t \rightarrow+\infty$, then $Y(t)$ gives a good approximation to $X(t)$ - this is proved using appropriate sub- and super-solutions for $u(t, x)$ that are based on $w(t, x)$.

The Fisher-KPP equation also appears in the theory of branching random walks and branching Brownian motion (BBM) [14]. Consider a BBM starting at $x=0$ at time $t=0$, and let $X_{1}(t), \ldots, X_{N_{t}}(t)$ be the descendants of the original particle at time $t$, arranged in increasing order:

$$
X_{1}(t) \leq X_{2}(t) \leq \cdots \leq X_{N_{t}}(t)
$$

Then, the probability distribution function of the maximum:

$$
v(t, x)=\mathbb{P}\left(X_{N_{t}}(t)>x\right),
$$

satisfies the Fisher-KPP equation

$$
v_{t}=\frac{1}{2} v_{x x}+v-v^{2}
$$

with the initial data $v_{0}(x)=\mathbb{I}_{x \leq 0}$. Recently, Fang and Zeitouni considered the asymptotics of the position of the maximum for a branching random walk with a time-dependent diffusivity: first, with a variance that takes just two values $\sigma_{1}$ and $\sigma_{2}$ on the time intervals $0 \leq t \leq T / 2$ and $T / 2 \leq t \leq T$ [4], and then for a monotonic in time variance of the form $\sigma(t / T)[5]$. They have observed that Bramson's correction $3 / 2 \log T$ for the front position $X(T)$ is valid only for constant diffusivities, and, moreover, for a monotonic in time variance the deviation is actually of the size $O\left(T^{1 / 3}\right)$, which is a completely different behavior. Their results are described in more detail in Sections 3 and 4.

The main result of the present paper (see Theorem 4.1) is a PDE proof (based on the aforementioned ideas from $[10,11])$ and a refinement of the results of Fang and Zeitouni. More precisely, we consider solutions of

$$
u_{t}=\sigma^{2}(t / T) u_{x x}+f(u),
$$

with front-like initial data $u_{0}(x)$. We show that if $\sigma(s)$ is increasing, the front position has the asymptotics

$$
X(T)=c_{e f f} T-\bar{\nu} T^{1 / 3}+O(\log T), \text { as } T \rightarrow+\infty .
$$


Here, the effective speed is

$$
c_{e f f}=2 \int_{0}^{1} \sigma(s) d s,
$$

and the explicit constant in front of $T^{1 / 3}$ is

$$
\bar{\nu}=\beta \int_{0}^{1} \sigma(\tau)^{1 / 3} \dot{\sigma}(\tau)^{2 / 3} d \tau,
$$

and $-\beta<0$ is the first zero of the Airy function $\operatorname{Ai}(x)$. We note that a crucial ingredient leading to the $T^{1 / 3}$ correction is the fact that $\dot{\sigma}(s)$ is positive - it leads to time variation in the exponential decay rate $\lambda(s)=1 / \sigma(s)$ that ultimately creates the balance in the Airy eigenvalue problem that is necessary to observe $T^{1 / 3}$-delay. We refer the reader to the proof of Theorem 4.1 for details of the balance.

When the diffusivity $\sigma^{2}$ depends on time, the relations (1.9) and (1.10) between the branching Brownian motion and the Fisher-KPP equation may be generalized as follows. Each of the branching particles satisfies

$$
d X(t)=\sqrt{2} \sigma(t) d B(t)
$$

where $B(t)$ is a standard Brownian motion in $\mathbb{R}$ (for each particle there is a different Brownian motion, independent of the others). Suppose there is one particle initially at time $s$ at position $y$. The particles branch independently at rate $\gamma>0$, and we label them $X_{M(t)} \leq \cdots \leq X_{2}(t) \leq X_{1}(t)$, so that $X_{M(t)}$ refers to the minimal particle at time $t$. From a modification of the arguments in [14], it follows that the function

$$
z(s, y ; t)=\mathbb{P}\left(X_{M(t)}>0 \mid X_{1}(s)=y\right)
$$

satisfies the terminal value problem

$$
z_{s}+\sigma^{2}(s) z_{y y}=\gamma z-\gamma z^{2}, \quad s<t, \quad y \in \mathbb{R}
$$

with the terminal condition being the indicator function

$$
z(t, y ; t)=\mathbb{I}_{(0, \infty)}(y)
$$

Therefore, the function

$$
v(r, y ; t)=1-z(t-r, y ; t)=\mathbb{P}\left(X_{M(t)} \leq 0 \mid X_{1}(t-r)=y\right)
$$

satisfies the initial value problem

$$
v_{r}=\sigma^{2}(t-r) v_{y y}+\gamma v(1-v), \quad y \in \mathbb{R}, \quad r>0
$$

with initial condition

$$
v(0, y ; t)=\mathbb{I}_{(-\infty, 0]}(y),
$$

which is in the class of initial value problems that were are studying. In particular, by comparing (1.13) and (1.14), we see that solutions of the Fisher-KPP equation (i.e. (1.11) with $f=u-u^{2}$ ) with a diffusion coefficient $\sigma^{2}$ that increases in time correspond to a BBM with diffusion coefficient $\sigma^{2}$ that decreases in time (as in $[4,5]$ ).

The paper is organized as follows. In Section 2 we recall what happens for the fully linearized problem. In Section 3 we review Fang's and Zeitouni's "two-variances" setting and explain how their results can be obtained by PDE-based computations. This leads us to the main theorem of this paper, Theorem 4.1, stated in Section 4. The last two sections are devoted to its proof. 
Throughout the paper we assume for simplicity of notation that $f^{\prime}(0)=1$, and denote by $C, C^{\prime}$ etc. various constants that do not depend on $T$.

Acknowledgment. We thank Ofer Zeitouni for illuminating and patient discussions. LR was supported by the NSF grant DMS-0908507 and NSF FRG grant DMS-115893. JN was supported by DMS-1007572. The research leading to these results has received funding from the European Research Council under the European Union's Seventh Framework Programme (FP/2007-2013)/ERC Grant Agreement n.321186 - ReaDi -Reaction-Diffusion Equations, Propagation and Modelling.

\section{2 "Front position" in the linear case}

It is instructive to first consider how solutions of the linearized equation

$$
\phi_{t}=\sigma^{2}(t) \phi_{x x}+\phi, \quad x \in \mathbb{R}, \quad t>0
$$

with compactly supported initial data $\phi(0, x)=\phi_{0}(x)$ spread on the whole real line as $t$ increases. We assume that $\phi_{0}(x) \in L^{1}(\mathbb{R})$ is nonnegative, compactly supported, and positive on a set of positive measure. The "front" position is defined in this setting as

$$
X_{\text {lin }}(t)=\sup \{x \in \mathbb{R}: \phi(t, x)=1\} .
$$

Note that, unlike the solution of the KPP equation, the solution of the linear problem is not bounded by 1 from above, and

$$
\lim _{t \rightarrow+\infty} \phi(t, x)=+\infty \text { for all } x \in \mathbb{R},
$$

so $X_{\text {lin }}(t)$, as defined in (2.2), is finite, at least for sufficiently large times (depending on the initial data). In order to find the front position, we make a time change

$$
s(t)=\int_{0}^{t} \sigma^{2}(r) d r
$$

Then the solution of (2.1) can be written as $\phi(t, x)=e^{t} v(s(t), x)$. Here, the function $v(s, x)$ solves the heat equation

$$
v_{s}=v_{x x}, \quad s>0,
$$

with the initial data $v(0, x)=\phi_{0}(x)$. The front position is, therefore, determined by

$$
e^{t} v\left(s(t), X_{\text {lin }}(t)\right)=\frac{e^{t}}{\sqrt{4 \pi s(t)}} \int_{\mathbb{R}} \exp \left\{-\frac{\left(X_{\text {lin }}(t)-y\right)^{2}}{4 s(t)}\right\} \phi_{0}(y) d y=1 .
$$

Without loss of generality, and to simplify the computations, suppose that the initial data $\phi_{0}$ is the characteristic function of an interval $[0, M]$ :

$$
\phi_{0}(x)=\mathbb{I}_{[0, M]}(x) .
$$

It is easy to see directly from (2.3) that $X_{\text {lin }}(t) \rightarrow+\infty$ as $t \rightarrow+\infty$. Hence the integral in (2.3) may be estimated as follows for large $t$ :

$$
\begin{aligned}
& \int_{0}^{M} \exp \left\{-\frac{\left(X_{\text {lin }}(t)-y\right)^{2}}{4 s(t)}\right\} d y=\int_{0}^{M} \exp \left\{-\frac{X_{\text {lin }}^{2}(t)-2 X_{\text {lin }}(t) y+y^{2}}{4 s(t)}\right\} d y \\
& =e^{-X_{\text {lin }}^{2}(t) / 4 s(t)} \int_{0}^{M} \exp \left\{\frac{X_{l i n}(t) y}{2 s(t)}\right\} d y\left[1+O\left(\frac{1}{s(t)}\right)\right] \\
& =e^{-X_{\text {lin }}^{2}(t) / 4 s(t)} \frac{2 s(t)}{X_{l i n}(t)}\left[e^{M X_{\text {lin }}(t) / 2 s(t)}-1\right]\left[1+O\left(\frac{1}{s(t)}\right)\right] .
\end{aligned}
$$


It is also straightforward to verify, using the maximum principle, that $X_{l i n}(t) / s(t)=O(1)$ as $t \rightarrow$ $+\infty$. Therefore, (2.3) and (2.4) show that

$$
\frac{e^{t}}{\sqrt{4 \pi s(t)}} e^{-X_{\operatorname{lin}}^{2}(t) / 4 s(t)}=O(1)
$$

that is:

$$
t-\frac{X_{l i n}^{2}(t)}{4 s(t)}-\frac{1}{2} \log s(t)=O(1)
$$

We deduce that

$$
\left.X_{\text {lin }}(t)=\left(4 s(t) t\left(1-\frac{\log s(t)}{2 t}+O\left(\frac{1}{t}\right)\right)\right)^{1 / 2}=2 \sqrt{s(t) t}\left(1-\frac{\log s(t)}{4 t}+O\left(\frac{1}{t}\right)\right)\right) .
$$

As $s(t) / t=O(1)$, it follows that the front position has the asymptotics

$$
X_{\text {lin }}(t)=2\left(\frac{1}{t} \int_{0}^{t} \sigma^{2}(\xi) d \xi\right)^{\frac{1}{2}} t-\frac{1}{2}\left(\frac{1}{t} \int_{0}^{t} \sigma^{2}(\xi) d \xi\right)^{\frac{1}{2}} \log t+O(1) .
$$

If we define the average variance as

$$
\sigma_{e f f}(t)=\left(\frac{1}{t} \int_{0}^{t} \sigma^{2}(\xi) d \xi\right)^{1 / 2}
$$

the front position can be written in a form similar to that in a homogeneous medium (c.f. (1.5)):

$$
X_{\text {lin }}(t)=2 \sigma_{\text {eff }}(t) t-\frac{\sigma_{e f f}(t)}{2} \log t+O(1), \quad \text { as } t \rightarrow \infty .
$$

That is, the front position lags by " $1 / 2 \log t$ " behind the "linear" in time asymptotics. Moreover, it depends only on the average variance $\sigma_{e f f}(t)$. For example, if $\sigma(t)$ has the form

$$
\sigma(t)= \begin{cases}\sigma_{1}, & 0 \leq t \leq T / 2 \\ \sigma_{2}, & T / 2<t \leq T\end{cases}
$$

then $X_{\text {lin }}(T)$ does not depend on whether $\sigma_{1}>\sigma_{2}$ or $\sigma_{2}>\sigma_{1}$. This is very different from what happens for the (nonlinear) KPP equation, where, as it was discovered by Fang and Zeitouni in [4], the front position depends very much on the relation between $\sigma_{1}$ and $\sigma_{2}$.

\section{KPP with a piece-wise constant diffusivity}

In order to appreciate the difference between the behavior of the solutions of the KPP equation and those of the linearized problem, we first recover from the PDE point of view the results of [4] obtained for the discrete version of

$$
\begin{aligned}
& u_{t}=\sigma^{2}(t) u_{x x}+u(1-u), \quad x \in \mathbb{R}, \quad t>0, \\
& u(0, x)=u_{0}(x), \quad x . \in \mathbb{R}
\end{aligned}
$$

Here, as in [4], the diffusivity $\sigma(t)$ has the form (2.11). We suppose that $0 \leq u_{0}<1$ and $u_{0}$ is compactly supported. As $u(t, x)$ satisfies $0<u(t, x)<1$, it is convenient to define the front position $X(t)$ by

$$
X(t)=\sup \{x \in \mathbb{R} \mid u(t, x)=1 / 2\}
$$


Let us define the averages

$$
\sigma_{a}=\frac{\sigma_{1}+\sigma_{2}}{2}
$$

and

$$
\sigma_{e}=\sqrt{\frac{\sigma_{1}^{2}+\sigma_{2}^{2}}{2}}
$$

The following proposition is a PDE version of the main result of [4].

Proposition 3.1 Let $u$ solve the nonlinear problem (3.1) where $\sigma(t)$ is the piece-wise constant function (2.11). If $\sigma_{1}<\sigma_{2}$, then we have

$$
X(T)=2 \sigma_{a} T-3 \sigma_{a} \ln T+O(1), \quad \text { as } T \rightarrow \infty .
$$

If $\sigma_{1}>\sigma_{2}$, then

$$
X(T)=2 \sigma_{e} T-\frac{1}{2} \sigma_{e} \ln T+O(1), \quad \text { as } T \rightarrow \infty .
$$

Comparing (3.5) and (3.6), we see that, in contrast to the linear case, both the speed and the logarithmic term are not invariant under a permutation of $\sigma_{1}$ and $\sigma_{2}$. The main observation of [4] is that while the delay behind the linear-in-time position is logarithmic in both cases, neither of the pre-factors $1 / 2$ and 3 is equal to the Bramson coefficient $3 / 2$ that appears when $\sigma(t)$ is constant in time - the case $\sigma_{1}=\sigma_{2}$ turns out to be degenerate. In [4] this result is stated in terms of a branching random walk. As we have explained in the introduction, this means that in Proposition 3.1, the case of increasing $\sigma$ (i.e. $\sigma_{1}<\sigma_{2}$ ) corresponds to decreasing $\sigma$ in the corresponding result in [4]. Similarly, decreasing $\sigma$ in Proposition 3.1 corresponds to increasing $\sigma$ in [4].

Since a probabilistic proof of Proposition 3.1 has been presented already in [4], we only outline the computations required for a PDE proof, and omit the details that can easily turn them into a rigorous proof. Let $c_{1}=2 \sigma_{1}$ be the minimal traveling wave speed associated with $\sigma_{1}$. That is, for any $c \geq c_{1}$ there is a traveling wave solution $v(t, x)=U_{c}(x-c t)$ to the equation

$$
v_{t}=\sigma_{1}^{2} v_{x x}+v(1-v)
$$

satisfying $U_{c}(+\infty)=0, U_{c}(-\infty)=1$. We denote by $U_{1}$ the wave profile corresponding to the minimal speed $c=c_{1}$, and by $\lambda_{1}=1 / \sigma_{1}$ the exponential decay of the wave: $U_{1}(x) \sim x e^{-\lambda_{1} x}$ as $x \rightarrow+\infty$. Similarly, $c_{2}=2 \sigma_{2}$, and $\lambda_{2}=1 / \sigma_{2}$ are the parameters associated with the minimial traveling wave solution for

$$
v_{t}=\sigma_{2}^{2} v_{x x}+v(1-v)
$$

\subsection{The front position when $\sigma_{1}<\sigma_{2}$}

We first look at the case $\sigma_{1}<\sigma_{2}$. For $0<t<T / 2$ the function $u(t, x)$ solves the KPP equation with a constant diffusivity $\sigma_{1}$. So, when $T$ is large, at the time $t=T / 2$ it will satisfy (see $[1,2,9,10,13,16]$ )

$$
u(T / 2, x) \leq U_{1}\left(x-c_{1} T / 2+\frac{3}{2 \lambda_{1}} \log (T / 2)+\delta_{1}\right) . \quad x \in \mathbb{R} .
$$

Here, the shift $\delta_{1}$ depends only on the initial data $u_{0}$. As the profile $U_{1}(y)$ has the asymptotics

$$
C_{1}^{-1} y e^{-\lambda_{1} y} \leq U_{1}(y) \leq C_{1} y e^{-\lambda_{1} y}, \quad y \geq 1,
$$

the function $u(T / 2, x)$ satisfies

$$
u(T / 2, x) \leq C_{1}\left(x-c_{1} T / 2+\frac{3}{2 \lambda_{1}} \log (T / 2)+\delta_{1}\right) \exp \left\{-\lambda_{1}\left(x-c_{1} T / 2+\frac{3}{2 \lambda_{1}} \log (T / 2)+\delta_{1}\right)\right\},
$$


for $x$ sufficiently large. Observe that, because $\sigma_{1}<\sigma_{2}$, the exponential decay rates satisfy $\lambda_{1}>\lambda_{2}$. In particular, for any $\lambda_{3} \in\left(\lambda_{2}, \lambda_{1}\right)$ we may choose $C_{2}$ so that

$$
u(T / 2, x) \leq \min \left(1, C_{2} \exp \left\{-\lambda_{3}\left(x-c_{1} T / 2+\frac{3}{2 \lambda_{1}} \log (T / 2)+\delta_{1}\right)\right\}\right), \quad x \in \mathbb{R} .
$$

Hence the function $u(T / 2, x)$ decays much faster than the critical traveling wave $U_{2}(x)$, and the evolution between the times $T / 2$ to $T$ is as if $u$ were compactly supported at time $T / 2$. In particular, it follows that

$$
X(T) \leq c_{1} \frac{T}{2}-\frac{3}{2 \lambda_{1}} \log (T / 2)+c_{2} \frac{T}{2}-\frac{3}{2 \lambda_{2}} \log (T / 2)+O(1) .
$$

Notice that this argument fails when $\sigma_{1}=\sigma_{2}$, and $\lambda_{1}=\lambda_{2}$.

A matching lower bound on $X(T)$ is obtained similarly. If $T$ is sufficiently large, then at the time $T / 2$ we have

$$
u(T / 2, x) \geq U_{1}\left(x-c_{1} T / 2+\frac{3}{2 \lambda_{1}} \log (T / 2)+\delta_{2}\right)-1 / 4, \quad x \geq 0,
$$

for some constant $\delta_{2}$ depending only on $u_{0}$. In particular, there is $\delta_{3}$ such that

$$
u\left(T / 2, x+c_{1} T / 2-\frac{3}{2 \lambda_{1}} \log (T / 2)+\delta_{3}\right) \geq \frac{1}{2} \mathbb{I}_{[0,1]}(x),
$$

for all $T$ sufficiently large. By the maximum principle, $u(T, x) \geq v(T, x)$ where

$$
\begin{aligned}
& v_{t}=\sigma_{2}^{2} v_{x x}+v(1-v), \quad t \geq T / 2, x \in \mathbb{R} \\
& v(T / 2, x)=\frac{1}{2} \mathbb{I}_{[0,1]}\left(x-c_{1} T / 2+\frac{3}{2 \lambda_{1}} \log (T / 2)-\delta_{3}\right), \quad x \in \mathbb{R} .
\end{aligned}
$$

The function $v(t, x)$ satisfies the KPP equation with a constant diffusivity $\sigma_{2}$, and its front position satisfies the "usual Bramson 3/2 $\log (T / 2)$ delay" after time $T / 2$ elapses for (3.9), which brings us to time $t=T$. Hence, the front position satisfies

$$
X(T) \geq c_{1} \frac{T}{2}-\frac{3}{2 \lambda_{1}} \log (T / 2)+c_{2} \frac{T}{2}-\frac{3}{2 \lambda_{2}} \log (T / 2)+O(1)
$$

Combining (3.8) and (3.10) we obtain

$$
X(T)=\left(c_{1}+c_{2}\right) T-\frac{3}{2}\left(\frac{1}{\lambda_{1}}+\frac{1}{\lambda_{2}}\right) \log T+O(1)
$$

which establishes (3.5).

\subsection{The front position when $\sigma_{1}>\sigma_{2}$}

Now, we consider the situation when $\sigma_{1}>\sigma_{2}$. The first task is compute the speed. We still have the same asymptotics for $u(t, x)$ at the time $t=T / 2$ :

$$
u(T / 2, x) \sim U_{1}\left(x-c_{1} T / 2\right) \approx\left\{\begin{array}{c}
1, x<c_{1} T / 2, \\
e^{-\lambda_{1}\left(x-c_{1} T / 2\right)-\left(x-c_{1} T / 2\right)^{2} /\left(4 \sigma_{1}^{2}(T / 2)\right)}, x>c_{1} T / 2,
\end{array}\right.
$$

which comes from solving the KPP equation with the diffusivity $\sigma_{1}$ on the time interval $[0, T / 2]$. Here, as we are computing only the speed, and not the precise asymptotics, the Bramson logarithmic 
correction was neglected. The key point is that the decay rate $\lambda_{1}$ is now slower than the decay rate $\lambda_{2}=1 / \sigma_{2}$ for the critical traveling wave associated with diffusivity $\sigma_{2}$. Solving the KPP equation with the diffusivity $\sigma_{2}$ for $T / 2 \leq t \leq T$, and with the initial data as in (3.11), at a time $t=s+T / 2$ we have, to the leading order:

$$
u\left(T / 2+s, c_{1} T / 2+x\right) \approx e^{s} \int_{0}^{\infty} e^{-(x-y)^{2} /\left(4 \sigma_{2}^{2} s\right)-\lambda_{1} y-y^{2} /\left(2 \sigma_{1}^{2} T\right)} d y
$$

and at $s=T / 2$ we get

$$
u\left(T, c_{1} T / 2+x\right) \approx e^{\frac{T}{2}-\frac{x^{2}}{2 \sigma_{2}^{2} T}} \int_{0}^{\infty} e^{-\frac{y^{2}}{2 \sigma_{2}^{2} T}+\frac{x y}{\sigma_{2}^{2} T}} e^{-\lambda_{1} y-\frac{y^{2}}{2 \sigma_{1}^{2} T}} d y .
$$

In order to find the speed, let us look for $\alpha>0$ such that $u\left(T, c_{1} T / 2+\alpha T\right)=O(1)$ :

$$
u\left(T, c_{1} T / 2+\alpha T\right) \approx \int_{0}^{\infty} e^{\frac{T}{2}-\frac{\alpha^{2} T}{2 \sigma_{2}^{2}}-\frac{y^{2}}{2 \sigma_{2}^{2} T}+\frac{\alpha y}{\sigma_{2}^{2}}} e^{-\lambda_{1} y-\frac{y^{2}}{2 \sigma_{1}^{2} T}} d y \approx e^{\frac{T}{2}-\frac{\alpha^{2} T}{2 \sigma_{2}^{2}}+\left(\frac{\alpha}{\sigma_{2}^{2}}-\lambda_{1}\right)^{2}\left(\frac{2}{\sigma_{2}^{2} T}+\frac{2}{\sigma_{1}^{2} T}\right)^{-1}}=O(1) .
$$

This gives

$$
1-\frac{\alpha^{2}}{\sigma_{2}^{2}}+\left(\frac{\alpha}{\sigma_{2}^{2}}-\frac{1}{\sigma_{1}}\right)^{2}\left(\frac{1}{\sigma_{2}^{2}}+\frac{1}{\sigma_{1}^{2}}\right)^{-1}=0,
$$

which after some algebra can reduced to

$$
\left(\alpha+\sigma_{1}\right)^{2}=2\left(\sigma_{1}^{2}+\sigma_{2}^{2}\right) .
$$

This gives the linear in time asymptotics for the front position

$$
X(T)=c_{1} \frac{T}{2}+\alpha T=\left(\sigma_{1}+\alpha\right) T=\sqrt{2\left(\sigma_{1}^{2}+\sigma_{2}^{2}\right)} T=2 \sigma_{e} T,
$$

as in (3.6).

Next, we outline how the above computation should be modified to compute the logarithmic shift. A more refined version of the asymptotics (3.11) for $u(T / 2, x)$ around the position $\xi(T)=c_{1} T / 2$ is

$$
u(T / 2, x) \geq(T / 2)^{-3 / 2}(x-\xi(T)) e^{-\lambda_{1}(x-\xi(T))-(x-\xi(T))^{2} /\left(4 \sigma_{1}^{2}(T / 2)\right)}, \quad x>\xi(T) .
$$

In order to find the logarithmic correction we need to take into account the factor of $(x-\xi(T))$ in front of the exponential in (3.14) (we did not need to account for it in the calculation of the speed, where (3.11) was sufficient). The "refined analog" of (3.12) for $t=s+T / 2$ is

$$
u(T / 2+s, \xi(T)+x) \approx T^{-3 / 2} \frac{e^{s}}{\sqrt{s}} \int_{0}^{\infty} y e^{-(x-y)^{2} /\left(4 \sigma_{2}^{2} s\right)-\lambda_{1} y-y^{2} /\left(2 \sigma_{1}^{2} T\right)} d y
$$

and at $s=T / 2$ we get

$$
u(T, \xi(T)+x) \approx \frac{1}{T^{2}} e^{\frac{T}{2}-\frac{x^{2}}{2 \sigma_{2}^{2} T}} \int_{0}^{\infty} y e^{-\frac{y^{2}}{2 \sigma_{2}^{2} T}+\frac{x y}{\sigma_{2}^{2} T}} e^{-\lambda_{1} y-\frac{y^{2}}{2 \sigma_{1}^{2} T}} d y .
$$

Taking $x=\alpha T+r \log T$, with $\alpha$ as in (3.13), and $r$ to be determined by the condition

$$
u\left(T, c_{1} T / 2+\alpha T+r \log T\right)=O(1),
$$


gives

$$
u(T, \xi(T)+\alpha T+r \log T) \approx \frac{1}{T^{2}} e^{\frac{T}{2}-\frac{\alpha^{2} T^{2}+2 \alpha r T \log T}{2 \sigma_{2}^{2} T}} \int_{0}^{\infty} y e^{-\frac{y^{2}}{2 \sigma_{2}^{2} T}+\frac{\alpha y}{\sigma_{2}^{2}}+\frac{r \log T}{\sigma_{2}^{2} T} y} e^{-\lambda_{1} y-\frac{y^{2}}{2 \sigma_{1}^{2} T}} d y=O(1) .
$$

Note that for $b \ll 1$ we have an approximation

$$
\int_{0}^{\infty} y e^{a y-b y^{2}} d y \approx \frac{a}{b^{3 / 2}} e^{a^{2} /(4 b)}
$$

Using this in (3.15) (in our case $b \sim 1 / T$ is small) gives

$$
\begin{aligned}
u(T, \xi(T)+\alpha T & +r \log T) \approx \frac{1}{T^{2}} e^{\frac{T}{2}-\frac{\alpha^{2} T}{2 \sigma_{2}^{2}}-\frac{\alpha r \log T}{\sigma_{2}^{2}}}\left(\frac{\alpha}{\sigma_{2}^{2}}-\lambda_{1}\right)\left(\frac{1}{2 \sigma_{2}^{2} T}+\frac{1}{2 \sigma_{1}^{2} T}\right)^{-3 / 2} \\
& \times \exp \left[\left(\frac{\alpha}{\sigma_{2}^{2}}+\frac{r \log T}{\sigma_{2}^{2} T}-\lambda_{1}\right)^{2} \frac{1}{4}\left(\frac{1}{2 \sigma_{2}^{2} T}+\frac{1}{2 \sigma_{1}^{2} T}\right)^{-1}\right]
\end{aligned}
$$

An elementary computation shows that the choice (3.13) of $\alpha$ cancels the terms in the exponent in (3.16) that are linear in $T$, while to cancel the terms multiplying $\log T$ in the exponent, we need to choose $r$ so that

$$
T^{-1 / 2} e^{-\alpha r \log T / \sigma_{2}^{2}} \exp \left[\left(\frac{\sigma_{1}^{2} \sigma_{2}^{2}}{\sigma_{1}^{2}+\sigma_{2}^{2}}\right) \frac{r \log T}{\sigma_{2}^{2}}\left(\frac{\alpha}{\sigma_{2}^{2}}-\lambda_{1}\right)\right]=1 .
$$

After some elementary transformations, this gives

$$
r=-\frac{1}{2} \sqrt{\frac{\sigma_{1}^{2}+\sigma_{2}^{2}}{2}}=-\frac{\sigma_{e}}{2},
$$

which is the logarithmic shift in (3.6). As we have mentioned, it is quite straightforward to make this argument rigorous using the precise asymptotics on the solutions of the KPP equation with constant coefficients from [10].

\section{The Fang-Zeitouni shift for an increasing diffusivity}

Motivated by the example of the variance that takes two different values as in Section 3, especially the case $\sigma_{1}<\sigma_{2}$, when the front delay is "surprisingly large", Fang and Zeitouni considered in [5] the following question: given a fixed time $T$ and all possible variances $\sigma(s)$, such that, say, $1 \leq \sigma(s) \leq 2$ for all $0 \leq s \leq T$, what is the largest possible KPP delay for compactly supported initial data? In order to make this question well-defined, they considered variances of the form $\sigma(s / T)$, with a smooth, bounded from above, and away from zero function $\sigma(s):[0,1]$, and studied solutions of

$$
\begin{aligned}
& u_{t}=\sigma^{2}(t / T) u_{x x}+f(u), \quad x \in \mathbb{R}, \quad t \in[0, T] \\
& u(0, x)=u_{0}(x), \quad x \in \mathbb{R},
\end{aligned}
$$

with compactly supported initial data $u_{0}(x)$, and with $f(u)=u-u^{2}$. We consider here more generally, a nonlinearity $f(u)$ of the KPP type, as in (1.2). The front position $X(t)$ for $0 \leq t \leq T$ is then defined as in (3.2).

Note that after rescaling $t=s T, x=y T$, we obtain the following form of (4.1):

$$
u_{s}=\delta \sigma^{2}(s) u_{y y}+\frac{1}{\delta} f(u)
$$


with $\delta=1 / T \ll 1$. The initial data for (4.2) is also rescaled: $u(s=0, y)=u_{0}(y / \delta)$ which creates some additional complications when $u_{0}$ is compactly supported. However, if we consider, for simplicity, the special initial data $u_{0}(x)=\mathbb{I}_{x \leq 0}$, as in the paper by Fang and Zeitouni, then it is invariant under rescaling. Using the analysis of Freidlin [7, 8] or Evans and Souganidis [3], it is straightforward to verify that when $\sigma(s)$ is increasing then for a step-function initial data the front speed is, asymptotically for large $T$ (see [5] for a detailed computation), given by

$$
\frac{X(T)}{T}=v_{\sigma}+o(1), \text { as } T \rightarrow+\infty,
$$

where

$$
v_{\sigma}=\int_{0}^{1} \sigma(s) d s
$$

We note that when $\sigma(s)$ is not strictly increasing in time, then the speed $v_{\sigma}$ need not be given by (4.4). For instance, when $\sigma(s)$ is decreasing in time, we have

$$
v_{\sigma}=\left(\int_{0}^{1} \sigma^{2}(s) d s\right)^{1 / 2},
$$

which is the same as the speed for the linearized problem (2.9).

The main result of Fang and Zeitouni in [5] is that when $\sigma(s)$ is increasing in time, the front position actually lags behind the linear-in-time asymptotics by a term of the order $T^{1 / 3}$ and not logarithmically:

$$
X(T)=v_{\sigma} T-g(T)
$$

with a function $g(T)$ that satisfies

$$
0<\liminf _{T \rightarrow+\infty} \frac{g(T)}{T^{1 / 3}} \leq \limsup _{T \rightarrow+\infty} \frac{g(T)}{T^{1 / 3}}<+\infty .
$$

The main result of the present paper is the following refinement of this asymptotics.

Theorem 4.1 Let $u$ satisfy (4.1) and define $X(t)$ by (3.2). Assume that $\dot{\sigma}(s)>0$ for $0 \leq s \leq 1$. Then

$$
X(T)=2 T \int_{0}^{1} \sigma(s) d s-\bar{\nu} T^{1 / 3}+O(\log T)
$$

as $T \rightarrow \infty$, where

$$
\bar{\nu}=\beta \int_{0}^{1} \sigma(\tau)^{1 / 3} \dot{\sigma}(\tau)^{2 / 3} d \tau, \quad 0 \leq s \leq 1,
$$

and $\beta>0$ is the the principal eigenvalue of the Airy operator

$$
-\varphi_{x x}+x \varphi(x)=\beta \varphi(x), \quad x>0 ; \quad \varphi(0)=0 .
$$

Note that the function $\hat{\varphi}(x)=\varphi(x+\beta)$ satisfies

$$
-\hat{\varphi}^{\prime \prime}(x)+x \hat{\varphi}(x)=0 \text { for } x \geq-\beta \text { with } \hat{\varphi}(-\beta)=0 .
$$

Therefore, $-\beta<0$ is the first zero of the Airy function $\operatorname{Ai}(x)$, and $\varphi(r)=c \operatorname{Ai}(r-\beta)$ for some constant $c>0$.

Unlike [5], the proof of this theorem is not probabilistic and relies on the techniques and ideas of $[10,11]$ that gave an alternative PDE proof of Bramson's delay in homogeneous and periodic 
media. As we have mentioned, the main idea is to consider a linear boundary value problem on a moving half line:

$$
\phi_{t}=\sigma^{2}(t / T) \phi_{x x}+\phi, \quad t \in[0, T], \quad x>Y(t)
$$

with the Dirichlet boundary condition $\phi(t, Y(t))=0$. The crucial observation is that when the moving boundary $Y(t)$ is appropriately chosen, solutions of the nonlinear KPP equation (4.1) and those of the linear problem (4.10) behave in a similar fashion. Roughly, if we choose $Y(t)$ to be too far on the left, solutions of (4.10) will grow exponentially in time, while if we take it too far to the right, they will decay exponentially in time. However, if we choose $Y(t)$ just right so that either solutions of (4.10) stay $O(1)$ in a certain region, or, at most, decay algebraically in time, then we can construct both sub- and super-solutions for the KPP equation (4.1) using the function $\phi(t, x)$, and use them to find the asymptotics for $X(T)$.

\section{Proof of Theorem 4.1: the upper bound}

\section{The Dirichlet moving boundary problem}

As we have mentioned, we will construct a sub-solution and a super-solution for the KPP equation (4.1) using the linearized problem (4.10) with the Dirichlet boundary condition on a moving boundary. Let $\phi(t, x) \geq 0$ satisfy (4.10) with $Y(t)$ to be specified later. The initial condition $\phi(0, x)=\phi_{0}(x)$ will also be chosen later on. Although $\phi$ clearly is a supersolution to the nonlinear equation, in the sense that

$$
\phi_{t} \geq \sigma^{2}(t / T) \phi_{x x}+\phi(1-\phi),
$$

we can not conclude that $u(t, x) \leq \phi(t, x)$ for $x>Y(t)$ provided that $u_{0}(x) \leq \phi_{0}(x)$ because of the boundary condition $\phi(t, Y(t))=0$, which $u$ does not satisfy. The remedy is to choose $Y(t)$ and $\phi_{0}(x) \geq u_{0}(x)$ so that for all $t \geq 0$ there exists a point where $Y_{1}(t)$ such that

$$
Y(t)<Y_{1}(t)<Y(t)+C \text {, and } \phi\left(t, Y_{1}(t)\right) \geq 1 .
$$

If this is the case, the function

$$
\bar{\phi}(t, x)=\left\{\begin{array}{cc}
1, & x \leq Y_{1}(t) \\
\min (1, \phi(t, x)), & x>Y_{1}(t)
\end{array}\right.
$$

is a true super-solution for the nonlinear problem in all of $\mathbb{R}$ as a minimum of two super-solutions. By the maximum principle, we conclude that $u(t, x) \leq \bar{\phi}(t, x)$ holds for all $t \in[0, T], x \in \mathbb{R}$, giving us an upper bound for $X(t)$ :

$$
X(t) \leq Z(t)=\sup \{x: \phi(t, x)=1 / 2\}
$$

Our task, therefore, includes getting good decay estimates on $\phi(t, x)$ so that we can control the location of $Z(t)$.

We claim that by choosing

$$
Y(t)=2 \int_{0}^{t} \sigma(\tau / T) d \tau-T^{1 / 3} l(t / T),
$$

with $l(s)$ defined by

$$
l(s)=\beta \int_{0}^{s} \sigma(\tau)^{1 / 3} \dot{\sigma}(\tau)^{2 / 3} d \tau, \quad 0 \leq s \leq 1,
$$


so that $\bar{\nu}=l(1)$, and with a suitable initial condition $\phi_{0} \geq 0$, the function $\phi(t, x)$ will satisfy

$$
\phi(t, x)=e^{-\lambda(t / T)(x-Y(t))} T^{1 / 3}\left[\varphi\left(\left(\frac{x-Y(t)}{T^{1 / 3}}\right) \frac{\dot{\sigma}(t / T)^{1 / 3}}{\sigma(t / T)^{4 / 3}}\right)+O\left(T^{-1 / 3}\right)\right]
$$

where $\varphi$ is the Airy eigenfunction in (4.9) and $\lambda(s)=1 / \sigma(s)$. More precisely, we will show that there is a constant $C$ such that for all $T>1$ there is a smooth function $h(s, r):[0,1] \times[0, \infty) \rightarrow \mathbb{R}$ such that

$$
\max _{0 \leq s \leq 1}\|h(s, \cdot)\|_{L^{\infty}([0, \infty))} \leq \frac{C}{T^{1 / 3}}
$$

and

$$
\phi(t, x)=e^{-\lambda(t / T)(x-Y(t))} T^{1 / 3}\left[\varphi\left(\left(\frac{x-Y(t)}{T^{1 / 3}}\right) \frac{\dot{\sigma}(t / T)^{1 / 3}}{\sigma(t / T)^{4 / 3}}\right)+h\left(\frac{t}{T^{1 / 3}},\left(\frac{x-Y(t)}{T^{1 / 3}}\right) \frac{\dot{\sigma}(t / T)^{1 / 3}}{\sigma(t / T)^{4 / 3}}\right)\right] .
$$

From above and the fact that $\varphi^{\prime}(0)>0$, we will conclude that, indeed, there is a continuous function $Y_{1}(t)$ such that (5.1) holds. In particular, we have

$$
u(T, x) \leq \bar{\phi}(T, x) \leq e^{-\lambda(1)(x-Y(T))} T^{1 / 3}\left[\varphi\left(\left(\frac{x-Y(T)}{T^{1 / 3}}\right) \frac{\dot{\sigma}(t / T)^{1 / 3}}{\sigma(t / T)^{4 / 3}}\right)+O\left(T^{-1 / 3}\right)\right], \quad x>Y_{1}(T) .
$$

It follows that

$$
X(T) \leq 2 T \int_{0}^{1} \sigma(s) d s-T^{1 / 3} l(1)+C \log T
$$

which is the required upper bound for $X(T)$ in Theorem 4.1.

\subsection{The problem in the moving frame}

Our goal is, therefore, to choose the initial data $\phi_{0}$ so that with $Y(t)$ given by (5.4) we would have (5.7) and (5.8), and $\phi_{0}(x) \geq u_{0}(x)$ for $x \geq 0$. To this end, let us consider $Y(t)$ of the form

$$
Y(t)=2 \int_{0}^{t} \sigma(\tau / T) d \tau-T^{m} l(t / T),
$$

with the exponent $m$ to be determined and $l(0)=0$. Shifting to the moving frame: $\phi(t, x)=$ $v(t, x-Y(t))$, we obtain

$$
v_{t}-2 \sigma(t / T) v_{y}+T^{m-1} \dot{l}(t / T) v_{y}=\sigma^{2}(t / T) v_{y y}+v, \quad y>0, \quad 0 \leq t \leq T,
$$

and $v(t, 0)=0$. The initial data is unchanged: $v(0, x)=\phi_{0}(x)$. Next, we take out the exponential decay (in space) factor: let $\lambda(s)=1 / \sigma(s)$, and define a function $w(s, y)=v(s T, y) e^{\lambda(s) y}$. A straightforward computation using the definition of $\lambda(s)$ shows that $w(s, y)$ satisfies

$$
\frac{1}{T} w_{s}+T^{m-1} \dot{l}(s) w_{y}=\sigma^{2}(s) w_{y y}+\left(T^{m-1} \frac{\dot{l}(s)}{\sigma(s)}-\frac{1}{T} \frac{\dot{\sigma}(s) y}{\sigma^{2}(s)}\right) w, \quad y>0, \quad 0 \leq s \leq 1,
$$

with the boundary condition $w(s, 0)=0$, and the initial condition $w(0, y)=e^{\lambda(0) y} \phi_{0}(y)$. After rescaling of the spatial variable: $y=T^{p} z$, this equation becomes

$$
\frac{1}{T} w_{s}+T^{m-p-1} \dot{l}(s) w_{z}=\sigma^{2}(s) T^{-2 p} w_{z z}+\left(T^{m-1} \frac{\dot{l}(s)}{\sigma(s)}-T^{p-1} \frac{\dot{\sigma}(s) z}{\sigma^{2}(s)}\right) w .
$$


The initial data in the rescaled variables is $w(0, z)=e^{\lambda(0) T^{p} z} \phi_{0}\left(T^{p} z\right)$.

Now, in order to balance the three terms in the right side of (5.10), we choose $m=p=1 / 3$, so that $-2 p=p-1=m-1$. Then, we have

$$
\varepsilon\left(w_{s}+\dot{l}(s) w_{z}\right)=\sigma^{2}(s) w_{z z}-\frac{\dot{\sigma}(s)}{\sigma^{2}(s)} z w+\frac{\dot{l}(s)}{\sigma(s)} w, \quad y>0,0<s \leq 1,
$$

with the initial data $w(0, z)=e^{\lambda(0) z / \varepsilon} \phi_{0}(z / \varepsilon)$, and the boundary condition $w(s, 0)=0$. Here we have set $\varepsilon=T^{-1 / 3}$.

We observe from (5.11) that if $\varepsilon$ is small, then $z \mapsto w(s, z)$ is almost a solution to the eigenvalue problem

$$
-\sigma^{2}(s) \bar{w}_{z z}+\frac{\dot{\sigma}(s)}{\sigma^{2}(s)} z \bar{w}=\mu(s) \bar{w}, \quad z>0 ; \quad \bar{w}(0)=0 .
$$

The shift $l(s)$ should be, therefore, related to the principal eigenvalue $\mu(s)$ for (5.12) via

$$
\mu(s)=i(s) / \sigma(s),
$$

which, after a simple rescaling of the eigenvalue problem, becomes (5.5). With this in mind, to simplify the eigenvalue problem, we rescale again by $z=r \sigma^{4 / 3}(\dot{\sigma})^{-1 / 3}$. Then in the variables $(s, r)$, (5.11) becomes

$$
\varepsilon \frac{\sigma^{2 / 3}}{\dot{\sigma}^{2 / 3}}\left(w_{s}+\left(\frac{\dot{\sigma}^{1 / 3}}{\sigma^{4 / 3}}\right)^{\prime} \frac{\sigma^{4 / 3}}{\dot{\sigma}^{1 / 3}} r w_{r}+\frac{\dot{l} \dot{\sigma}^{1 / 3}}{\sigma^{4 / 3}} w_{r}\right)=w_{r r}-r w+\frac{i}{\sigma^{1 / 3} \dot{\sigma}^{2 / 3}} w, \quad r>0
$$

with $w(s, 0)=0$, and the initial data

$$
w(0, r)=e^{\lambda(0) r \sigma^{4 / 3}(0)(\dot{\sigma})^{-1 / 3}(0) / \varepsilon} \phi_{0}\left(r \sigma^{4 / 3}(0)(\dot{\sigma})^{-1 / 3}(0) / \varepsilon\right) .
$$

\subsection{The small $\varepsilon$ asymptotics}

We now consider the small $\varepsilon$ behavior of the solutions of (5.13), where $\varepsilon=T^{-1 / 3}$. Recall that we denote by $\beta>0$ and $\varphi(r) \geq 0$, respectively, the principal eigenvalue and eigenfunction for the Airy operator

$$
\mathcal{L}_{r} \varphi=-\varphi_{r r}(r)+r \varphi(r)=\beta \varphi(r), \quad r>0 ; \quad \varphi(0)=0 .
$$

Here, $\varphi$ is normalized so that

$$
\int_{0}^{\infty} \varphi^{2} d r=1
$$

The operator $\mathcal{L}_{r}$ is self-adjoint on the space

$$
\mathcal{V}=\left\{\left.w \in H_{0}^{1}([0, \infty))\left|\int_{0}^{\infty} x\right| w(x)\right|^{2} d x<\infty\right\}
$$

We now choose the shift $l(s)$ to satisfy

$$
\dot{l}(s)=\beta \sigma(s)^{1 / 3} \dot{\sigma}(s)^{2 / 3}, \quad l(0)=0 .
$$

We also denote the coefficients

$$
k_{1}(s)=\frac{\beta \dot{\sigma}}{\sigma}, \quad k_{2}(s)=\frac{\dot{\sigma}^{2 / 3}}{\sigma^{2 / 3}}, \quad k_{3}(s)=\left(\frac{\dot{\sigma}^{1 / 3}}{\sigma^{4 / 3}}\right)^{\prime}\left(\frac{\sigma^{4 / 3}}{\dot{\sigma}^{1 / 3}}\right) .
$$


These are all bounded above and bounded away from zero. With this notation, equation (5.13) for $w(s, r)$ becomes

$$
w_{s}=\varepsilon^{-1} k_{2}(s)\left(\beta-\mathcal{L}_{r}\right) w-k_{1}(s) w_{r}-k_{3}(s) r w_{r}, \quad r>0, \quad s \in[0,1] .
$$

We will prove the following:

Lemma 5.1 There is a constant $C$ such that if $w(s, r)$ solves (5.17) with $w(s, 0)=0$ and initial condition $w(0, r)=\varphi(r)$, then

$$
\max _{0 \leq s \leq 1}\|w(s, \cdot)-\varphi(\cdot)\|_{L^{\infty}([0, \infty))} \leq C \varepsilon
$$

This lemma implies the upper bound in Theorem 4.1. Indeed, $\phi(t, x)$ is related to $w(s, r)$ by

$$
\phi(t, x)=e^{-\lambda(t / T)(x-Y(t))} w\left(\frac{t}{T},\left(\frac{x-Y(t)}{T^{1 / 3}}\right) \frac{\dot{\sigma}(t / T)^{1 / 3}}{\sigma(t / T)^{4 / 3}}\right) .
$$

We then choose the initial condition $\phi_{0}$ so that $w(0, r)=T^{1 / 3} \varphi(r)$ :

$$
\phi_{0}(x)=e^{-\lambda(0) x} T^{1 / 3} \varphi\left(\frac{x}{T^{1 / 3}} \frac{\dot{\sigma}(0)^{1 / 3}}{\sigma(0)^{4 / 3}}\right) .
$$

Having chosen $\phi_{0}$ by (5.18), Lemma 5.1 implies that

$$
\left\|w(s, \cdot)-T^{1 / 3} \varphi(\cdot)\right\|_{L^{\infty}} \leq C,
$$

which implies (5.7) and (5.8).

Next, we ensure that initially we have $\phi_{0}(x) \geq u_{0}(x)$ for $x \geq 0$. Since $\varphi^{\prime}(0)>0$, for any $M>0$ we have

$$
\phi_{0}(x) \geq e^{-\lambda(0) x} T^{1 / 3} \varphi\left(\frac{x}{T^{1 / 3}} \frac{\dot{\sigma}(0)^{1 / 3}}{\sigma(0)^{4 / 3}}\right) \geq\left(\frac{\dot{\sigma}(0)^{1 / 3}}{\sigma(0)^{4 / 3}} \frac{\varphi^{\prime}(0)}{2}\right) x e^{-\lambda(0) x}, \quad x \in[0, M],
$$

if $T$ is large enough. Hence, by multiplying $\phi_{0}(x)$ by another large constant (independent of $T$ ), we may guarantee that $\phi_{0}(x) \geq u_{0}(x)$, the latter being compactly supported on an interval $[0, M]$. Now, by the $L^{\infty}$ bound in Lemma 5.1 and since $\varphi^{\prime}(0)>0$, there are constants $C>0$ and $\rho>0$ such that for any $y \in\left[0, T^{1 / 3}\right]$

$$
\begin{aligned}
T^{1 / 3}[\varphi & \left.\left(\left(\frac{y}{T^{1 / 3}}\right) \frac{\dot{\sigma}(t / T)^{1 / 3}}{\sigma(t / T)^{4 / 3}}\right)+h\left(\frac{t}{T^{1 / 3}},\left(\frac{y}{T^{1 / 3}}\right) \frac{\dot{\sigma}(t / T)^{1 / 3}}{\sigma(t / T)^{4 / 3}}\right)\right] . \\
& \geq T^{1 / 3} \rho \frac{y}{T^{1 / 3}}-T^{1 / 3}\|h\|_{\infty} \geq T^{1 / 3} \rho \frac{y}{T^{1 / 3}}-T^{1 / 3} C T^{-1 / 3}=\rho y-C .
\end{aligned}
$$

Hence, taking $y_{0}>2 C / \rho$ sufficiently large (independent of $T$ ), we have

$$
\begin{aligned}
\phi\left(t, Y(t)+y_{0}\right) & =e^{-\lambda(t / T)\left(y_{0}\right)} T^{1 / 3}\left[\varphi\left(\left(\frac{y_{0}}{T^{1 / 3}}\right) \frac{\dot{\sigma}(t / T)^{1 / 3}}{\sigma(t / T)^{4 / 3}}\right)+h\left(\frac{t}{T^{1 / 3}},\left(\frac{y_{0}}{T^{1 / 3}}\right) \frac{\dot{\sigma}(t / T)^{1 / 3}}{\sigma(t / T)^{4 / 3}}\right)\right] \\
& \geq e^{-\lambda(t / T)\left(y_{0}\right)} \frac{\rho}{2} y_{0},
\end{aligned}
$$

for all $t \in[0, T]$. Therefore, by multiplying $\phi$ by a large constant, independent of $T$, we may guarantee that $\phi\left(t, Y(t)+y_{0}\right)>1$ holds for all $t \in[0, T]$. In particular, we may set $Y_{1}(t)=Y(t)+y_{0}$ and define $\bar{\phi}(t, x)$ as in (5.2) so that $\bar{\phi}(t, x) \geq u(t, x)$ for all $x \in \mathbb{R}$ and $t \in[0, T]$. As explained above, this establishes the upper bound (5.9), except for the proof of Lemma 5.1. 


\subsection{Proof of Lemma 5.1}

Let $w(s, r)=\alpha(s) \varphi(r)+\widetilde{w}(s, r)$, where $\langle\widetilde{w}, \varphi\rangle=0$ for all $s$, and define

$$
\delta(s)=\int_{0}^{\infty} \widetilde{w}^{2} d r \geq 0 .
$$

First, we will show that for some constant $C$ and $\rho>0$, independent of $\varepsilon$,

$$
\|w(s, \cdot)-\alpha(0) \varphi(\cdot)\|_{L^{2}([0, \infty))} \leq C e^{-\rho \varepsilon^{-1} s / 2} \delta(0)^{1 / 2}+C \varepsilon\left(\alpha(0)+\delta^{1 / 2}(0)\right) .
$$

holds for all $s \in[0,1]$. Then, we bootstrap this $L^{2}$-bound to an $L^{\infty}$ estimate.

Let $M$ and $A$ denote the operators

$$
M w=k_{2}(s)\left(\beta-\mathcal{L}_{r}\right) w,
$$

and

$$
A w=-k_{1}(s) w_{r}-k_{3}(s) r w_{r} .
$$

Multiplying (5.17) by $w$ and integrating by parts we have

$$
\begin{aligned}
\frac{1}{2} \frac{d}{d s} \int_{0}^{\infty} w^{2} d r & =\varepsilon^{-1} k_{2}(s)\left\langle\left(\beta-\mathcal{L}_{r}\right) w, w\right\rangle-k_{1}(s)\left\langle w, w_{r}\right\rangle-k_{3}(s)\left\langle r w_{r}, w\right\rangle \\
& =\varepsilon^{-1} k_{2}(s)\left\langle\left(\beta-\mathcal{L}_{r}\right) w, w\right\rangle+\frac{k_{3}(s)}{2}\langle w, w\rangle \leq \frac{k_{3}(s)}{2}\langle w, w\rangle .
\end{aligned}
$$

Therefore, by Gronwall's inequality we have

$$
\int_{0}^{\infty} w^{2}(s, r) d r \leq e^{K} \int_{0}^{\infty} w^{2}(0, r) d r
$$

for all $s \in[0,1]$, where $K=\max _{0 \leq \tau \leq 1} \int_{0}^{\tau} k_{3}(s) d s$. In particular, we have an a priori bound on $\alpha(s)$ :

$$
\begin{aligned}
|\alpha(s)|=\left|\int_{0}^{\infty} w(s, r) \varphi(r) d r\right| & \leq e^{K / 2}\left(\int_{0}^{\infty} w^{2}(0, r) d r\right)^{1 / 2} \\
& =e^{K}\left(\alpha^{2}(0)+\delta(0)\right)^{1 / 2} \leq e^{K}\left(\alpha(0)+\delta^{1 / 2}(0)\right),
\end{aligned}
$$

and on $\delta(s)$ :

$$
0 \leq \delta(s)=\int_{0}^{\infty}(\widetilde{w}(s, r))^{2} d r \leq e^{K} \int_{0}^{\infty} w^{2}(0, r) d r=e^{K}\left(\alpha^{2}(0)+\delta(0)\right)
$$

that hold for all $s \in[0,1]$, since $\widetilde{w}$ and $\varphi$ are orthogonal. Using (5.17), we also compute

$$
\begin{aligned}
& \delta^{\prime}(s)=\frac{d}{d s} \int_{0}^{\infty} \widetilde{w}^{2}(s, r) d r=\varepsilon^{-1}\langle\widetilde{w}, M \widetilde{w}\rangle+\langle\widetilde{w}, A w\rangle=-\varepsilon^{-1} k_{2}(s) \int_{0}^{\infty}\left(\left|\widetilde{w}_{r}\right|^{2}+(r-\beta) \widetilde{w}^{2}\right) d r \\
& -k_{1}(s) \alpha(s) \int_{0}^{\infty} \widetilde{w}(s, r) \varphi^{\prime}(r) d r-k_{3}(s) \int_{0}^{\infty} \widetilde{w}(s, r) r w_{r}(s, r) d r \\
& \leq-\varepsilon^{-1} k_{2}(s)\left(\beta_{2}-\beta\right) \int_{0}^{\infty} \widetilde{w}^{2} d r-k_{1}(s) \alpha(s) \int_{0}^{\infty} \widetilde{w}(s, r) \varphi^{\prime}(r) d r-k_{3}(s) \int_{0}^{\infty} \widetilde{w}(s, r) r w_{r}(s, r) d r \\
& =-\varepsilon^{-1} k_{2}(s)\left(\beta_{2}-\beta\right) \int_{0}^{\infty} \widetilde{w}^{2} d r-k_{1}(s) \alpha(s) \int_{0}^{\infty} \widetilde{w}(s, r) \varphi^{\prime}(r) d r \\
& +\frac{k_{3}(s)}{2} \int_{0}^{\infty} \widetilde{w}^{2} d r-k_{3}(s) \alpha(s) \int_{0}^{\infty} \widetilde{w} r \varphi^{\prime} d r
\end{aligned}
$$


where $\beta_{2}>\beta$ is the second eigenvalue of the Airy operator $\left(-\beta_{2}<-\beta<0\right.$ is the second root of the Airy function $\operatorname{Ai}(x))$. Since

$$
\min _{0 \leq s \leq 1} k_{2}(s)>0
$$

we have

$$
k_{3}(s) \leq \varepsilon^{-1} k_{2}(s)\left(\beta_{2}-\beta\right) \text { for all } s \in[0,1],
$$

if $\varepsilon$ is small enough. Hence, (5.25) implies

$$
\delta^{\prime}(s) \leq-\varepsilon^{-1} \frac{k_{2}(s)}{2}\left(\beta_{2}-\beta\right) \int_{0}^{\infty} \widetilde{w}^{2} d r-k_{1}(s) \alpha(s) \int_{0}^{\infty} \widetilde{w}(s, r) \varphi^{\prime}(r) d r-k_{3}(s) \alpha(s) \int_{0}^{\infty} \widetilde{w} r \varphi^{\prime} d r .
$$

Let

$$
I_{1}(s)=\int_{0}^{\infty} \widetilde{w} \varphi^{\prime} d r, \quad I_{3}(s)=\int_{0}^{\infty} r \widetilde{w} \varphi^{\prime} d r .
$$

By the Cauchy-Schwarz inequality, we have

$$
\left|I_{1}(s)\right|^{2} \leq \delta(s) \int_{0}^{\infty}\left(\varphi^{\prime}\right)^{2} d r
$$

By definition of $\varphi$ and its normalization, we have

$$
\int_{0}^{\infty}\left(\varphi^{\prime}\right)^{2} d r \leq \beta
$$

So

$$
\left|I_{1}(s)\right|^{2} \leq \delta(s) \beta
$$

Similarly, we have

$$
\left|I_{3}(s)\right|^{2} \leq C \delta(s) \int r^{2}\left(\varphi^{\prime}\right)^{2} d r .
$$

Using this and (5.23) and (5.24) in (5.26) we obtain

$$
\delta^{\prime}(s) \leq-\varepsilon^{-1} \rho \delta(s)-\alpha(s) k_{1}(s) I_{1}(s)-k_{3}(s) \alpha(s) I_{3}(s) \leq-\varepsilon^{-1} \rho \delta(s)+C|\alpha(s)| \delta(s)^{1 / 2},
$$

for all $s \in[0,1]$, for some constant $\rho>0$. This gives

$$
\left(e^{\rho \varepsilon^{-1} s} \delta(s)\right)^{\prime} \leq C|\alpha(s)| \delta(s)^{1 / 2} e^{\rho \varepsilon^{-1} s}=C|\alpha(s)|\left(e^{\rho \varepsilon^{-1} s} \delta(s)\right)^{1 / 2} e^{\rho \varepsilon^{-1} s / 2},
$$

which, in turn, implies

$$
\left(e^{\rho \varepsilon^{-1}} t \delta(t)\right)^{1 / 2} \leq\left(e^{\rho \varepsilon^{-1} 0} \delta(0)\right)^{1 / 2}+\frac{C}{2} \int_{0}^{t}|\alpha(s)| e^{\rho \varepsilon^{-1} s / 2} d s .
$$

Therefore, using (5.23), we conclude that

$$
\begin{aligned}
0 \leq \delta(t)^{1 / 2} & \leq e^{-\rho \varepsilon^{-1} t / 2} \delta(0)^{1 / 2}+\frac{C}{2} \int_{0}^{t}|\alpha(s)| e^{-\rho \varepsilon^{-1}(t-s) / 2} d s \\
& \leq e^{-\rho \varepsilon^{-1} t / 2} \delta(0)^{1 / 2}+C \varepsilon\left(\alpha(0)+\delta^{1 / 2}(0)\right) .
\end{aligned}
$$

Now we estimate $\alpha^{\prime}(s)$. Returning to (5.17) again, we obtain

$$
\begin{aligned}
\frac{d}{d s} \alpha(s) & =\frac{d}{d s} \int_{0}^{\infty} \varphi(r) w(s, r) d r=\varepsilon^{-1}\langle\varphi, M w\rangle+\langle\varphi, A w\rangle=\langle\varphi, A w\rangle \\
& =k_{1}(s) \int_{0}^{\infty} \varphi^{\prime}(r) w(s, r) d r+k_{3}(s) \int_{0}^{\infty}\left(\widetilde{w} \varphi+\widetilde{w} r \varphi^{\prime}\right) d r \\
& =k_{1}(s) \int_{0}^{\infty} \varphi^{\prime}(r) \widetilde{w}(s, r) d r+k_{3}(s) \int_{0}^{\infty} \widetilde{w} r \varphi^{\prime} d r .
\end{aligned}
$$


Therefore, by Cauchy-Schwarz and (5.29), we have

$$
\left|\alpha^{\prime}(s)\right| \leq C \sqrt{\delta(s)} \leq C e^{-\rho \varepsilon^{-1} t / 2} \delta(0)^{1 / 2}+C \varepsilon\left(\alpha(0)+\delta^{1 / 2}(0)\right), \quad \forall s \in[0,1] .
$$

Since $\varphi$ and $\widetilde{w}$ are orthogonal and $\|\varphi\|_{2}=1$, we now combine 5.29) and (5.31) to conclude that

$$
\begin{aligned}
\|w(s, \cdot)-\alpha(0) \varphi(\cdot)\|_{L^{2}([0, \infty))} & \leq\|w(s, \cdot)-\alpha(s) \varphi(\cdot)\|_{L^{2}([0, \infty))}+|\alpha(s)-\alpha(0)| \\
& =\delta^{1 / 2}(s)+|\alpha(s)-\alpha(0)| \\
& \leq C \sqrt{\delta(s)} \leq C e^{-\rho \varepsilon^{-1} t / 2} \delta(0)^{1 / 2}+C \varepsilon\left(\alpha(0)+\delta^{1 / 2}(0)\right)
\end{aligned}
$$

holds for all $s \in[0,1]$. This proves (5.21).

Now we strengthen (5.21) to obtain an $L^{\infty}$ estimate. The function $h(s, r)=w(s, r)-\alpha(0) \varphi(r)$ satisfies

$$
h_{s}=\varepsilon^{-1} k_{2}(s)\left(\beta-\mathcal{L}_{r}\right) h-k_{1}(s) h_{r}-k_{3}(s) r h_{r}-k_{1}(s) \alpha(0) \varphi_{r}-k_{3}(s) \alpha(0) r \varphi_{r} .
$$

Changing variables $\tau=\varepsilon^{-1} s$, leads to

$$
h_{\tau}=k_{2}(\varepsilon \tau) h_{r r}+k_{2}(\varepsilon \tau)(\beta h-r h)-\varepsilon k_{1}(\varepsilon \tau) h_{r}-\varepsilon k_{3}(\varepsilon \tau) r h_{r}-\varepsilon k_{1}(\varepsilon \tau) \alpha(0) \varphi_{r}-\varepsilon k_{3}(s) \alpha(0) r \varphi_{r} .
$$

The coefficients $k_{1}, k_{2}, k_{3}$ are smooth and bounded independently of $\varepsilon$ and $k_{2}>0$ is bounded away from zero. The functions $\varphi_{r}$ and $r \varphi_{r}$ are also smooth and bounded. Therefore, by considering the Cauchy problem starting at a time $\tau_{1}$ on the time interval $\tau_{1} \leq \tau \leq \tau_{1}+1$ with the initial data $h\left(\tau_{1}, r\right)$, we see that the maximum principle implies that there is a constant $C^{\prime}$ such that for any $\tau_{1} \in\left[0, \varepsilon^{-1}-1\right]$,

$$
\max _{\tau \in\left[\tau_{1}, \tau_{1}+1\right]}\|h(\tau, \cdot)\|_{L^{\infty}} \leq C^{\prime}\left\|h\left(\tau_{1}, \cdot\right)\right\|_{L^{\infty}}+C^{\prime} \varepsilon \alpha(0) .
$$

From (5.21), we also know that

$$
\int_{0}^{\infty}|h(\tau, r)|^{2} d r<C \gamma(\tau, \varepsilon)^{2} \quad \text { for all } \tau \in\left[0, \varepsilon^{-1}\right] .
$$

where

$$
\gamma(\tau, \varepsilon)=C e^{-\rho \tau} \delta(0)^{1 / 2}+C \varepsilon\left(\alpha(0)+\delta^{1 / 2}(0)\right) .
$$

Now, let $\tau_{0}$ be any time in the interval $\left[0, \varepsilon^{-1}\right]$. Multiply (5.34) by $h$ and integrate:

$$
\frac{1}{2} \frac{d}{d t} \int_{0}^{\infty} h^{2} d r \leq-c_{0} \int_{0}^{\infty} h_{r}^{2} d r+C \beta \int_{0}^{\infty} h^{2} d r+C \varepsilon \int_{0}^{\infty} h^{2} d r+C \varepsilon \alpha(0)\|h\|_{L^{2}} .
$$

Integrating in time and using the bound (5.36), we obtain

$$
c_{0} \int_{\tau_{0}}^{\tau_{0}+1} \int_{0}^{\infty} h_{r}^{2}(\tau, r) d r d \tau \leq C \gamma\left(\tau_{0}, \varepsilon\right)^{2}+C \varepsilon \alpha(0) \gamma\left(\tau_{0}, \varepsilon\right)
$$

Therefore, there exists a time $\tau_{1} \in\left[\tau_{0}, \tau_{0}+1\right]$ for which

$$
\int_{0}^{\infty} h_{r}^{2}\left(\tau_{1}, r\right) d r \leq C \gamma\left(\tau_{0}, \varepsilon\right)^{2}+C \varepsilon \alpha(0) \gamma\left(\tau_{0}, \varepsilon\right)
$$

Hence, at this time $\tau_{1}$ we have

$$
\int_{0}^{\infty} h_{r}^{2}\left(\tau_{1}, r\right) d r+\int_{0}^{\infty} h^{2}\left(\tau_{1}, r\right) d r \leq C \gamma\left(\tau_{0}, \varepsilon\right)^{2}+C \varepsilon \alpha(0) \gamma\left(\tau_{0}, \varepsilon\right),
$$


and thus

$$
\left\|h\left(\tau_{1}, \cdot\right)\right\|_{L^{\infty}(0,+\infty)} \leq C \gamma\left(\tau_{0}, \varepsilon\right)+C \sqrt{\varepsilon \alpha(0) \gamma\left(\tau_{0}, \varepsilon\right)} .
$$

Since $\tau_{0}$ was chosen arbitrarily and since $\tau_{0}+1 \in\left[\tau_{1}, \tau_{1}+1\right]$, we combine this with (5.35) to conclude that

$$
\left\|h\left(\tau_{0}+1, \cdot\right)\right\|_{L^{\infty}(0,+\infty)} \leq C^{\prime \prime} \gamma\left(\tau_{0}, \varepsilon\right)+C \sqrt{\varepsilon \alpha(0) \gamma\left(\tau_{0}, \varepsilon\right)}+C^{\prime \prime} \varepsilon \alpha(0), \quad \forall \tau_{0} \in\left[0, \varepsilon^{-1}-1\right] .
$$

For $\tau \in[0,1],(5.35)$ also implies that

$$
\|h(\tau, \cdot)\|_{L^{\infty}} \leq C^{\prime \prime}\|h(0, \cdot)\|_{L^{\infty}}+C^{\prime \prime} \varepsilon \alpha(0), \quad \forall \tau \in[0,1] .
$$

If $\delta(0)=0$, then $\gamma(\tau, \varepsilon) \leq C \varepsilon \alpha(0)$ for all $\tau \geq 0$. Furthermore, $h(0, \cdot) \equiv 0$ in this case. Therefore, the combination of (5.41) and (5.42) implies that

$$
\|h(\tau, \cdot)\|_{L^{\infty}} \leq C \varepsilon \alpha(0)
$$

holds for all $\tau \in\left[0, \varepsilon^{-1}\right]$. This concludes the proof of the lemma.

\section{Proof of the lower bound in Theorem 4.1}

\subsection{Outline of the proof}

Step 1. We will, once again, use the linearized problem with the Dirichlet boundary condition on a moving boundary. However, the solution of

$$
\phi_{t}=\sigma^{2}(t / T) \phi_{x x}+f^{\prime}(0) \phi, \quad x>Y(t), \quad \phi(t, Y(t)=0,
$$

would not be a true sub-solution for the nonlinear problem since $f^{\prime}(0) u \geq f(u)$. Therefore, we assume without loss of generality that $f^{\prime}(0)=1$, and replace $f(u)$ by the linear function $f_{1}(u)=\left(1-T^{-1 / 10}\right) u$ so that $f(u) \geq f_{1}(u)$ for all $u \leq T^{-1 / 10}$. The idea is to choose $Y(t)$ so that until the time $T$ the solution $\psi(t, x)$ to this modified linear problem stays below $T^{-1 / 10}$. By our choice of $f_{1}$ this makes it a sub-solution for the nonlinear equation. To this end, we will set

$$
Y_{s}(t)=2 \int_{0}^{t} \sigma(s / T) d s-T^{1 / 3} l(t / T)
$$

with $l(t)$ almost as in the proof of the upper bound except we will end up with

$$
l(1)=l_{\text {old }}(1)+\frac{C \log T}{T^{1 / 3}} .
$$

Given $Y_{s}(t)$, we choose a function $\psi_{0}(x) \leq u_{0}(x)$ such that the solution of

$$
\psi_{t}=\sigma^{2}(t / T) \psi_{x x}+\left(1-T^{-10}\right) \psi, \quad x>Y_{s}(t)
$$

with the initial condition $\psi(0, x)=\psi_{0}(x)$ and the boundary condition $\psi\left(t, Y_{s}(t)\right)=0$ satisfies the following two conditions: first,

$$
\psi(t, x) \leq T^{-10}, \text { for all } 0 \leq t \leq T \text { and all } x>Y_{s}(t),
$$

and, second,

$$
\psi\left(t, Y_{s}(t)+1\right) \geq T^{-100}, \text { for all } T / 2 \leq t \leq T
$$


It follows that $u(t, x) \geq \psi(t, x)$ for all $0 \leq t \leq T$ and $x \geq Y_{s}(t)$. The difficulty in this step is to make sure that (6.1)-(6.3) are all compatible.

Step 2. The main consequence of Step 1 is that the solution of the nonlinear problem

$$
u_{t}=\sigma^{2}(t / T) u_{x x}+f(u)
$$

satisfies a lower bound

$$
u\left(t, Y_{s}(t)+1\right) \geq T^{-100} \text { for all } T / 2 \leq t \leq T .
$$

Let us assume that the initial data $u_{0}(x)$ is front-like and monotonic: if not, we can bound it from below by a monotonic function. Then $u_{x}(t, x)<0$ for all $t>0$ and $x \in \mathbb{R}$, whence $u(t, x) \geq T^{-100}$ for all $t \in[T / 2, T]$ and $x \leq Y_{s}(t)+1$.

Let $\tau=T-C \log T$ and consider the KPP equation

$$
v_{t}=\sigma^{2}(1) v_{x x}+f(v), \quad \tau \leq t \leq T,
$$

on a fixed interval $I_{L}=\left[Y_{s}(\tau)-2 L, Y_{s}(\tau)-L\right]$, with the Dirichlet boundary conditions

$$
v\left(t, Y_{s}(\tau)-2 L\right)=v\left(t, Y_{s}(\tau)-L\right)=0, \quad \tau \leq t \leq T,
$$

and with the initial data $v(\tau, x)=T^{-100} \leq u(\tau, x)$. As the function $v(t, x)$ is concave in $x$ for all $\tau \leq t \leq T$, and the function $\sigma(s)$ is increasing, it satisfies

$$
v_{t} \leq \sigma^{2}(t / T) v_{x x}+f(v), \quad \tau \leq t \leq T, \quad x \in I_{L} .
$$

Then $u(t, x) \geq v(t, x)$ for all $x \in I_{L}$ and $\tau \leq t \leq T$. Let us choose $L$ sufficiently large (independent of $T$ ) so that there exists a steady solution $\bar{v}(x)$ of $(6.6)$ :

$$
\begin{aligned}
& \sigma^{2}(1) \bar{v}_{x x}+f(\bar{v})=0, \\
& \bar{v}\left(t, Y_{s}(\tau)-2 L\right)=v\left(t, Y_{s}(\tau)-L\right)=0 .
\end{aligned}
$$

It follows that $v(t, x)$ converges to $\bar{v}(x)$ exponentially fast as $t-\tau \rightarrow \infty$. More precisely, we have the following estimate:

$$
|v(t, x)-\bar{v}(x)| \leq C^{\prime} T^{100} e^{-c_{1}(t-\tau)} .
$$

Obviously, by taking the constant $C$ in the definition of $\tau$ sufficiently large we can guarantee that the exponential term $e^{-c_{1}(T-\tau)}$ is much smaller than the term $T^{100}$ in (6.8), whence

$$
u(T, x) \geq \bar{v}(x)-\frac{1}{T}
$$

for all $x \in I_{L}$. In particular, $u(T, x) \geq \max _{y \in I_{L}} \bar{v}(y)-\frac{1}{T}$ holds for all $x \leq Y_{s}(\tau)-2 L$, which implies that $X(T) \geq Y_{s}(T)-C^{\prime \prime} \log T$. Therefore, to complete the proof of the lower bound on $X(T)$, we only need to verify that (6.1)-(6.3) are compatible.

\subsection{Verification of the claim of Step 1}

Let us proceed as in the proof of the upper bound: we start with

$$
\psi_{t}=\sigma^{2}(t / T) \psi_{x x}+\left(1-T^{-10}\right) \psi, \quad x>Y_{s}(t), \quad t>0,
$$


with $\psi\left(t, Y_{s}(t)\right)=0$, and set

$$
Y_{s}(t)=2 \int_{0}^{t} \sigma(\tau / T) d \tau-T^{1 / 3} l(t / T),
$$

with $l(s)$ to be determined. Making the change of variables

$$
\psi(t, x)=v\left(t, x-Y_{s}(t)\right)
$$

gives

$$
v_{t}-2 \sigma(t / T) v_{y}+T^{-2 / 3} \dot{i}(t / T) v_{y}=\sigma^{2}(t / T) v_{y y}+\left(1-T^{-10}\right) v
$$

Next, we write

$$
v(t, y)=e^{-\lambda(t / T) y} \widetilde{w}(t / T, y)
$$

to get

$$
\begin{aligned}
& \frac{1}{T} \widetilde{w}_{s}-\frac{y}{T} \dot{\lambda}(s) \widetilde{w}-2 \sigma(s) \widetilde{w}_{y}+2 \sigma(s) \lambda(s) \widetilde{w}+T^{-2 / 3} \dot{i}(s) \widetilde{w}_{y}-T^{-2 / 3} i(s) \lambda(s) \widetilde{w} \\
& =\sigma^{2}(s) \widetilde{w}_{y y}-2 \lambda(s) \sigma^{2}(s) \widetilde{w}_{y}+\left[\sigma^{2}(s) \lambda^{2}(s)+1-T^{-10}\right] \widetilde{w} .
\end{aligned}
$$

We choose $\lambda(s)=1 / \sigma(s)$, as before, so that $\widetilde{w}(s, y)$ satisfies

$$
\frac{1}{T} \widetilde{w}_{s}+T^{-2 / 3} \dot{i}(s) \widetilde{w}_{y}=\sigma^{2}(s) \widetilde{w}_{y y}+\left[T^{-2 / 3} \frac{\dot{l}(s)}{\sigma(s)}-T^{-10}-\frac{y \dot{\sigma}(s)}{T \sigma^{2}(s)}\right] \widetilde{w},
$$

with the initial data $\widetilde{w}(0, y)=v(0, y) e^{\lambda(0) y}=\psi(0, y) e^{\lambda(0) y}$.

The next step is to write $\widetilde{w}(s, y)=w\left(s, y / T^{1 / 3}\right) e^{-s / T^{9}}$ :

$$
\frac{1}{T} w_{s}+\frac{1}{T} \dot{l}(s) w_{z}=\frac{1}{T^{2 / 3}} \sigma^{2}(s) w_{z z}+\left[T^{-2 / 3} \frac{\dot{l}(s)}{\sigma(s)}-\frac{z \dot{\sigma}(s)}{T^{2 / 3} \sigma^{2}(s)}\right] w
$$

with $w(0, z)=\widetilde{w}\left(0, T^{1 / 3} z\right)=\psi\left(0, T^{1 / 3} z\right) e^{\lambda(0) T^{1 / 3} z}$, and the boundary condition $w(t, 0)=0$. Setting $\varepsilon=T^{-1 / 3}$ gives, as before:

$$
\varepsilon\left[w_{s}+\dot{l}(s) w_{z}\right]=\sigma^{2}(s) w_{z z}+\left[\frac{\dot{l}(s)}{\sigma(s)}-z \dot{\sigma}(s) \sigma^{2}(s)\right] w,
$$

with $w(0, z)=\psi(0, z / \varepsilon) e^{\lambda(0) z / \varepsilon}$, and the boundary condition $w(t, 0)=0$. As in the construction of the super-solution, we set $z=r \sigma^{4 / 3}(\dot{\sigma})^{-1 / 3}$, so that (6.9) becomes

$$
\varepsilon \frac{\sigma^{2 / 3}}{\dot{\sigma}^{2 / 3}}\left(w_{s}+\left(\frac{\dot{\sigma}^{1 / 3}}{\sigma^{4 / 3}}\right)^{\prime} \frac{\sigma^{4 / 3}}{\dot{\sigma}^{1 / 3}} r w_{r}+\frac{i \dot{\sigma}^{1 / 3}}{\sigma^{4 / 3}} w_{r}\right)=w_{r r}-r w+\frac{i}{\sigma^{1 / 3} \dot{\sigma}^{2 / 3}} w, \quad r>0
$$

with $w(s, 0)=0$, and $w(0, r)=e^{\lambda(0) r \sigma^{4 / 3}(0)(\dot{\sigma})^{-1 / 3}(0) / \varepsilon} \psi_{0}\left(r \sigma^{4 / 3}(0)(\dot{\sigma})^{-1 / 3}(0) / \varepsilon\right)$. Then we choose $l(s)$ to satisfy

$$
\dot{l}(s)=\beta \sigma(s)^{1 / 3} \dot{\sigma}(s)^{2 / 3},
$$

so that with the previous notation for $k_{1,2,3}(s)$ we get

$$
w_{s}=\varepsilon^{-1} k_{2}(s)\left(\beta-\mathcal{L}_{r}\right) w-k_{1}(s) w_{r}-k_{3}(s) r w_{r}, \quad r>0, \quad s \in[0,1] .
$$


In summary, we have

$$
\psi\left(t, x+Y_{s}(t)\right)=e^{-\lambda(t / T) y} w\left(\frac{t}{T}, \frac{x}{T^{1 / 3}} \frac{\dot{\sigma}(t / T)^{1 / 3}}{\sigma(t / T)^{4 / 3}}\right) e^{-t / T^{10}}
$$

where $w(s, r)$ satisfies (6.11), which is the same equation as before (i.e. (5.17)). The difference here is that initial data for $\psi$ is compactly supported, say, in the interval $[0,1]$, in order that $\psi(0, x) \leq u_{0}(x)$. This means that we may choose the initial data for $w$ in the form

$$
w(0, r)=\varepsilon^{\eta} \mathbb{I}_{[0, N]}\left(\frac{r}{\varepsilon}\right)
$$

where $\eta>0$ will be chosen later. In particular, the initial data $w(0, r)$ cannot be bounded from below by a multiple of the eigenfunction $\varphi$.

With initial condition (6.12), let us decompose $w$ as in the proof of Lemma 5.1: $w(s, r)=$ $\alpha(s) \varphi(r)+\widetilde{w}(s, r)$ where $\langle\varphi(\cdot), \widetilde{w}(s, \cdot)\rangle=0$ for all $s$. Since $\varphi^{\prime}(0)>0$, we see that

$$
\alpha(0)=\int_{0}^{\infty} w(0, r) \varphi(r) d r=O\left(\varepsilon^{\eta} \varepsilon^{2}\right) .
$$

Also, let $\delta(s)=\int_{0}^{\infty} \widetilde{w}^{2}(s, r) d r$, so that

$$
\delta(0)=\int_{0}^{\infty}(w(0, r)-\alpha(0) \varphi(r))^{2} d r=O\left(\varepsilon^{2 \eta} \varepsilon\right) .
$$

The computations in Lemma 5.1 still apply. In particular, (5.41) and (5.42) shows that

$$
\|w(s, \cdot)\|_{L^{\infty}(0, \infty)} \leq\|w(s, \cdot)-\alpha(0) \varphi(\cdot)\|_{L^{\infty}(0, \infty)}+\|\alpha(0) \varphi\|_{L^{\infty}(0, \infty)} \leq C \varepsilon^{\eta}
$$

holds for all $s \in[0,1]$ and $\varepsilon \in(0,1]$. This implies the uniform upper bound on $\psi$ :

$$
\psi\left(t, x+Y_{s}(t)\right) \leq C \varepsilon^{\eta}=C T^{-\eta / 3}, \quad \forall x \geq 0, \quad t \in[0, T]
$$

which is (6.2), assuming we fix $\eta>30$.

Now we derive the lower bound (6.3) through a lower bound on $w$. The problem is that the projection of $w(0, r)$ onto the eigenfunction $\varphi(r)$ is relatively small compared to its projection onto the orthogonal complement of $\varphi$ (i.e. $\alpha(0)=O\left(\varepsilon^{\eta+2}\right)$, while $\delta^{1 / 2}(0)=O\left(\varepsilon^{\eta+1 / 2}\right)$ ). To address this issue, we first show that there exists a constant $C>0$ so that

$$
w(s=\varepsilon, r) \geq C \varepsilon^{\eta+2}, \text { for all } r \in[1,2] .
$$

To see why (6.14) must hold, we set $\tau=s / \varepsilon$ and to avoid dealing with unbounded coefficients we note that solution of (6.11) is bounded from below by the solution of the Dirichlet problem

$$
\bar{w}_{\tau}=k_{2}(\varepsilon \tau)\left(\beta-\mathcal{L}_{r}\right) \bar{w}-k_{1}(\varepsilon \tau) \bar{w}_{r}-k_{3}(\varepsilon \tau) r \bar{w}_{r}, \quad 0<r<10, \quad \tau \in[0,1],
$$

with the boundary condition $\bar{w}(\tau, 0)=\bar{w}(\tau, 10)=0$ and the initial condition $\bar{w}(0, r)=w(0, r)$ given by (6.12). As all coefficients are now bounded, the function $\bar{w}(s, r)$ for $\tau \in[0,1]$ is, up to a constant factor, and a bounded time change, of the same order as the solution of

$$
\bar{v}_{\tau}=\bar{v}_{r r}-\widetilde{k}_{1}^{\varepsilon}(\tau) \bar{v}_{r}-\widetilde{k}_{3}^{\varepsilon}(\tau) r \bar{v}_{r}, \quad 0<r<10, \quad \tau \in[0,1],
$$


also with the zero boundary conditions and the same initial data, and appropriate, uniformly bounded $\widetilde{k}_{1,3}^{\varepsilon}(t)$. Hence, it suffices to show that $\bar{v}(s=1, r) \geq C \varepsilon^{\eta+2}$ for all $r \in[1,2]$. Let us denote

$$
b(\tau, r)=-\widetilde{k}_{1}^{\varepsilon}(\tau)-\widetilde{k}_{3}^{\varepsilon}(\tau) r
$$

so that our problem is

$$
\bar{v}_{\tau}=\bar{v}_{r r}+b(\tau, r) \bar{v}_{r}
$$

Let us denote

$$
B(\tau, r)=\int_{0}^{r} b(\tau, x) d x
$$

The function $\eta(\tau, r)=e^{B(\tau, r) / 2} \bar{v}(\tau, r)$ satisfies

$$
\eta_{\tau}=\eta_{r r}+\left(\frac{B_{\tau}}{2}-\frac{B_{r r}}{2}-\frac{B_{r}^{2}}{4}\right) \eta
$$

So, if

$$
M=\max _{\substack{\tau \in[0,1] \\ r \in[0,10]}}\left(\frac{|B|}{2}+\frac{\left|B_{\tau}\right|}{2}+\frac{\left|B_{r r}\right|}{2}+\frac{\left|B_{r}\right|^{2}}{4}\right)
$$

we have $\bar{v}(\tau, r)=e^{-B / 2} \eta(\tau, r) \geq e^{-M} \bar{\rho}(\tau, r)$ for all $\tau \in[0,1]$ and $r \in[0,10]$, where $\bar{p}(\tau, r)$ solves the heat equation

$$
\bar{p}_{\tau}=\bar{p}_{r r}, \quad 0<r<10, \quad \tau \in[0,1],
$$

with the zero Dirichlet boundary conditions and $\bar{p}(0, r)=w(0, r)$ given by (6.12). In particular, $\bar{w}(\tau, r) \geq C \bar{p}(\tau, r)$. A simple computation shows that

$$
\bar{p}(\tau=1, r) \geq C \varepsilon^{\eta+2} \text { for all } r \in[1,2] .
$$

We conclude that (6.14) holds.

As a consequence of (6.14), the function $w(s, r)$ satisfies the lower bound $w(s, r) \geq C \varepsilon^{\eta+2} q(s, r)$ for $s>\varepsilon$, where $q$ is the solution of

$$
q_{s}=\varepsilon^{-1} k_{2}(s)\left(\beta-\mathcal{L}_{r}\right) q-k_{1}(s) q_{r}-k_{3}(s) r q_{r}, \quad r>0, \quad s \in[\varepsilon, 1],
$$

with the Cauchy data

$$
q(\varepsilon, r)= \begin{cases}1, & 1 \leq r \leq 2 \\ 0, & \text { otherwise }\end{cases}
$$

As we have done before, let us decompose $q(s, r)$ as

$$
q(s, r)=\alpha(s) \varphi(r)+\widetilde{q}(s, r)
$$

where $\langle\widetilde{q}(s, \cdot), \varphi(\cdot)\rangle=0$ for all $s$ and let

$$
\delta(s)=\int_{0}^{\infty} \widetilde{q}^{2}(s, r) d r .
$$

Observe that, at the initial time $s=\varepsilon$, we now have $C_{1} \leq \alpha(\varepsilon) \leq C_{2}$ and $C_{1} \leq \delta(\varepsilon) \leq C_{2}$ for some constant $C_{1}, C_{2}>0$, independent of $\varepsilon$. That is, the projection of $q(\varepsilon, r)$ onto $\varphi$ and its orthogonal complement are comparable in magnitude. The computations in Lemma 5.1 now apply to $q(s, r)$ (with the initial time at $s=\varepsilon$ rather than $s=0$ ). In particular, (5.41) and (5.42) shows that

$$
\|q(s, \cdot)-\alpha(\varepsilon) \varphi(\cdot)\|_{L^{\infty}(0, \infty)} \leq C \varepsilon\left(\alpha(\varepsilon)+\delta^{1 / 2}(\varepsilon)\right) \leq C_{3} \varepsilon
$$


holds for all $s \in[1 / 2,1]$. Therefore,

$$
w(s, r) \geq C \varepsilon^{\eta+2} q(s, r) \geq C \varepsilon^{\eta+2}\left(\alpha(\varepsilon) \varphi(r)-C_{3} \varepsilon\right)
$$

holds for all $r \geq 0, s \geq 1 / 2$. Choosing $r=j \varepsilon$, with $j$ fixed, and using the fact that $\varphi^{\prime}(0)>\rho>0$, we have

$$
w(s, j \varepsilon) \geq C_{4} \varepsilon^{\eta+3} \rho j-C_{5} \varepsilon^{\eta+3}
$$

for $s \geq 1 / 2$, if $\varepsilon$ is small enough, depending on $j$. So, if we fix $j$ large enough, this is bounded below by $C_{6} \varepsilon^{\eta+3}>0$ once $\varepsilon$ is small enough. Putting this in terms of $\psi$, we have

$$
\psi\left(t, j+Y_{s}(t)\right) \geq C w\left(\frac{t}{T}, j \varepsilon \frac{\dot{\sigma}(t / T)^{1 / 3}}{\sigma(t / T)^{4 / 3}}\right) \geq C \varepsilon^{\eta+3}=C T^{-(\eta+3) / 3}
$$

for all $t \in[T / 2, T]$. The Harnack inequality and the fact that $\psi \geq 0$ now implies that $\psi\left(t, 1+Y_{s}(t)\right) \geq$ $C^{\prime} T^{-(\eta+3) / 3}$ also holds for all $t \in[T / 2, T]$. In view of this and (6.13), we see that (6.3) and (6.2) hold if $\eta=50$, for example. This completes the proof of the lower bound in Theorem 4.1.

\section{References}

[1] M.D. Bramson, Maximal displacement of branching Brownian motion, Comm. Pure Appl. Math. 31, 1978, 531-581.

[2] M.D. Bramson, Convergence of solutions of the Kolmogorov equation to travelling waves, Mem. Amer. Math. Soc. 44, 1983.

[3] L.C. Evans and P. Souganidis, A PDE Approach to Geometric Optics for Certain Semilinear Parabolic Equations, Indiana Univ. Math. J. 38, 1989, 141-172.

[4] M. Fang and O. Zeitouni, Branching random walks in time inhomogeneous environments, Electron. Jour. Prob. 17, 2012, 18pp.

[5] M. Fang and O. Zeitouni, Slowdown for time inhomogeneous branching Brownian motion. J. Stat. Phys. 149, 2012, 1-9.

[6] R.A. Fisher, The wave of advance of advantageous genes, Ann. Eugenics 7, 1937, 353-369.

[7] M. Freidlin, Functional Integration and Partial Differential Equations, Ann. Math. Stud., 109, Princeton University Press, 1985.

[8] M. Freidlin, Limit theorems for large deviations and reaction-diffusion equations, SIAM Jour. Appl. Math. 46, 1986, 639-675.

[9] J. Gärtner, Location of wave fronts for the multi-dimensional K-P-P equation and Brownian first exit densities, Math. Nachr. 105, 1982, 317-351.

[10] F. Hamel, J. Nolen, J.-M. Roquejoffre and L. Ryzhik, A short proof of the logarithmic Bramson correction in Fisher-KPP equations, Networks and Heterogeneous Media 8, 2013, 275-289.

[11] F. Hamel, J. Nolen, J.-M. Roquejoffre and L. Ryzhik, The logarithmic delay of KPP fronts in a periodic medium, Preprint, 2012, arxiv:1211.6173 
[12] A.N. Kolmogorov, I.G. Petrovsky and N.S. Piskunov, Étude de l'équation de la diffusion avec croissance de la quantité de matière et son application à un problème biologique, Bull. Univ. État Moscou, Sér. Inter. A 1, 1937, 1-26.

[13] K.-S. Lau, On the nonlinear diffusion equation of Kolmogorov, Petrovskii and Piskunov, J. Diff. Eqs. 59, 1985, 44-70.

[14] H.P. McKean, Application of Brownian motion to the equation of Kolmogorov-PetrovskiiPiskunov, Comm. Pure Appl. Math. 28 (1975), 323-331.

[15] M. Roberts, A simple path to asymptotics for the frontier of a branching Brownian motion, Ann. Prob., to appear.

[16] K. Uchiyama, The behavior of solutions of some nonlinear diffusion equations for large time, J. Math. Kyoto Univ. 18, 1978, 453-508. 